\title{
Macromolecular Modelling and Docking Simulations for the Discovery of Selective GPER Ligands
}

\author{
Camillo Rosano, ${ }^{1,4}$ Marco Ponassi, ${ }^{1}$ Maria Francesca Santolla, ${ }^{2}$ Assunta Pisano, ${ }^{2}$ Lamberto Felli, ${ }^{3}$ \\ Adele Vivacqua, ${ }^{2}$ Marcello Maggiolini, ${ }^{2,4}$ and Rosamaria Lappano ${ }^{2}$
}

Received 26 September 2015; accepted 5 November 2015; published online 16 November 2015

\begin{abstract}
Estrogens influence multiple physiological processes and are implicated in many diseases as well. Cellular responses to estrogens are mainly mediated by the estrogen receptors (ER) $\alpha$ and $\mathrm{ER} \beta$, which act as ligand-activated transcription factors. Recently, a member of the G proteincoupled receptor (GPCR) superfamily, namely GPER/GPR30, has been identified as a further mediator of estrogen signalling in different pathophysiological conditions, including cancer. Today, computational methods are commonly used in all areas of health science research. Among these methods, virtual ligand screening has become an established technique for hit discovery and optimization. The absence of an established three-dimensional structure of GPER promoted studies of structure-based drug design in order to build reliable molecular models of this receptor. Here, we discuss the results obtained through the structure-based virtual ligand screening for GPER, which allowed the identification and synthesis of different selective agonist and antagonist moieties. These compounds led significant advances in our understanding of the GPER function at the cellular, tissue, and organismal levels. In particular, selective GPER ligands were critical toward the evaluation of the role elicited by this receptor in several pathophysiological conditions, including cancer. Considering that structure-based approaches are fundamental in drug discovery, future research breakthroughs with the aid of computer-aided molecular design and chemobioinformatics could generate a new class of drugs that, acting through GPER, would be useful in a variety of diseases as well as in innovative anticancer strategies.
\end{abstract}

KEY WORDS: drug design; GPCRs; GPER; molecular modelling; structural bioinformatics.

\section{INTRODUCTION}

Estrogens, which are natural-occurring cyclopentanophenanthrene moieties deriving from cholesterol, play a key role in different pathophysiological processes (1). Estrogens mainly act by binding to and activating the estrogen receptors $(E R) \alpha$ and $E R \beta$ that upon nuclear translocation regulate the transcription of target genes (1). As estrogens elicit rapid effects $(2,3)$, it has been suggested that proteins different from the classical ER can also mediate the multifaceted estrogen action. In this context, a seven-transmembrane-spanning G protein-coupled receptor (GPCR), namely GPER or GPR30, was identified and widely characterized toward its involvement in estrogen signalling $(4,5)$. The ligand activation of

\footnotetext{
${ }^{1}$ UOS Proteomics IRCCS AOU San Martino- IST National Institute for Cancer Research, Largo R. Benzi 10, 16132, Genoa, Italy.

${ }^{2}$ Department of Pharmacy, Health and Nutritional Sciences, University of Calabria, via Bucci, 87036, Rende, Cosenza, Italy.

${ }^{3}$ Department of Orthopedical Surgery, University of Genoa, Largo R. Benzi 10, 16132, Genoa, Italy.

${ }^{4}$ To whom correspondence should be addressed. (e-mail: marcellomaggiolini@yahoo.it)
}

GPER stimulates a transduction network which includes the epidermal growth factor receptor (EGFR), crucial intracellular pathways like phosphatidylinositol 3-kinase/protein kinase $\mathrm{B}$ (PI3K/Akt), mitogen-activated protein kinase/extracellular regulated protein kinase (MAPK/ERK), calcium mobilization, and others in both normal and malignant cells (6). Notably, GPER activation triggers a peculiar gene signature involved in cancer cell growth, migration, and angiogenesis (6-9). In accordance with these findings, previous studies have associated GPER expression with negative clinical features and poor survival rates in a variety of tumors (1012). As it concerns ligand activation, many compounds bind to ER and GPER in a promiscuous manner displaying either an agonist action through both receptors or an opposite activity. For instance, the ER antagonist 4-hydroxytamoxifen showed the ability to act as a GPER agonist (6). In recent years, various selective GPER ligands have been discovered, and the characterization of their binding modes was analyzed by docking simulations or further computational approaches (13). Although no GPER atomic structure is deposited to date in the Protein Data Bank (PDB), increasing progress in protein modelling techniques has allowed the building of GPER models enabling docking simulations at the atomic- 
scale level. Data obtained are of considerable interest in order to design novel compounds that can better address the molecular mechanisms involved in the biological processes mediated by GPER.

\section{A COMPUTATIONAL APPROACH TO THE GPER 3D STRUCTURE: "FROM SEQUENCE TO CONSEQUENCE"}

The ability to visualize and manipulate the threedimensional structure of a macromolecule on a personal computer dramatically improved our understanding of the molecular mechanisms involved in the biological functions of living organisms. The availability of the spatial coordinates for each single atom of a protein can allow a precise description of different phenomena such as the catalysis mediated by an enzyme, the description of the intermediates of a biochemical reaction, or the binding mode of a receptor to cognate ligand(s).

There is a plethora of different techniques that may support the process of determining a protein structure. Up to date, the PDB contains more than 102,000 protein structures among which more than 30,000 are from human genes. The atomic coordinates available to date in PDB were almost always determined by X-ray crystallography; however, approximately $1 \%$ of the solved structures was determined by NMR (14). X-ray crystallography is one of the most accurate measurement techniques; nevertheless, it is not always possible to crystallize all molecules. Only a small fraction of the structures of GPCRs has been solved so far (15). Although more than 900 GPCR genes are known (16), just 40 encoded proteins have been crystallized and their structures solved. It is particularly difficult to crystallize GPCRs as they are membrane proteins, and in solution, they can adopt different flexible conformations. In addition, a bottleneck to successfully assess a GPCR atomic structure is the availability of the sample; in this regard, it is challenging to get a high quantity of a pure protein folded in a correct manner. Anyway, the limited number of GPCR structures solved up to now is not sufficient to cover the big structural diversity among the members of this family of receptors. Hence, the efforts of pharmaceutical companies and researchers are currently focused on reliable molecular models in order to elucidate the mechanisms that regulate the different ligandreceptor binding modes. The essential prerequisite for a correct and effective process of rational design of novel protein ligands is the availability of the three-dimensional atomic coordinates of the target. In this context, molecular modelling (MM) has become an essential technique to be employed when no experimentally derived atomic coordinates of the protein target are available or when it is necessary to target a complex of two or more proteins whose structures are known (17).

A three-dimensional (3D) model of a target can be built taking into account that different proteins with a similar primary sequence may show a comparable structure. This approach is strongly dependent on the quality of the protein sequence alignment, in particular the presence of "gaps" as well as the degree of similarity between the "sequence query" and the "sequence template." When the compared proteins share more than $50 \%$ of the amino acid's identity, the model has a good quality; on the other hand, it is not considered trustworthy if the identity of two compared primary structures is less than $25 \%(18,19)$. However, the studies of computational biology have allowed the process of model building to be more reliable even in the presence of a low degree of similarity between the query and the template structure, as illustrated for GPCRs $(20,21)$.

To date, diverse web services that can predict a 3D model of a protein primary structure are available. Among these, the most popular for building a three-dimensional model of GPCRs is the GPCR I-TASSER service (15). It allowed the building of a database containing the 3D models of 907 GPCR gene products of the human genome (http://cssb.biology.gatech.edu/skolnick/files/ gpcr/gpcr.html). However, the single structures deposited within this database may not be precise due to the difficulties in modelling the exposed loop regions, which display significant structural differences among the numerous receptors. These errors in the positioning of the various loops may lead to functional differences and therefore are critical for a reliable process of drug design. To improve the quality of the model, a "supervised" approach is mandatory; for instance, experimental details like mutational results, structure-activity relationships (SAR), and other functional information would improve the quality of a "machine-built" model.

GPER has not been successfully crystallized so far; therefore, all the rational approaches to design novel ligands have been carried out using a three-dimensional molecular model as a target. Certain models of GPER were obtained by a web server for 3D-structure prediction such as I-TASSER and then refined by extensive MD simulations (22,23); in addition, virtual and biomolecular screening taking advantage of models deposited in appropriate data banks were employed as well. Using the aforementioned approaches, several different natural and synthetic ligands of GPER (Fig. 1) have been identified along with their binding modes as resulting from docking simulations (13,24-35).

The primary structure of GPER has a high degree of homology with the chemokine GPCR subfamily; however, different chemokines tested did not trigger GPER-mediated action (36). In contrast, it has been demonstrated that some estrogens, phyto-xenoestrogens, and antiestrogens can bind to GPER, which then activates downstream transduction pathways (37). Structurally, GPER is composed by 375 amino acid residues arranged in a seven-helical bundle intramembrane fold that is typical for all GPCRs. A conserved proline residue induces a kink in the seventransmembrane (TM) helices TM-I, TM-V, TM-VI, and TMVII. These kinks seem to be involved in the rearrangements that govern the activation of the $\mathrm{G}$ proteins. The $\mathrm{N}$-terminal fragment of GPER points toward the outside of the membrane and is composed by 60 residues (Met 1 to Phe 60) organized as a small glycosylated domain. The C-terminal of the protein is protruding inwards the membrane that, similarly to the proteins of the rhodopsin family, forms a further helical structure (residues Thr 330-Lys 342) followed by a "disordered coil." The seven-transmembrane helices, typical of the GPCR family members, are connected by extracellular and intracellular loops (EL and IL, respectively). While ELs may play a role in substrate recognition and binding, ILs are responsible for the binding of heterotrimeric $G$ proteins and the activation of intracellular transduction pathways. In addition, two cysteine residues, Cys 130 and Cys 


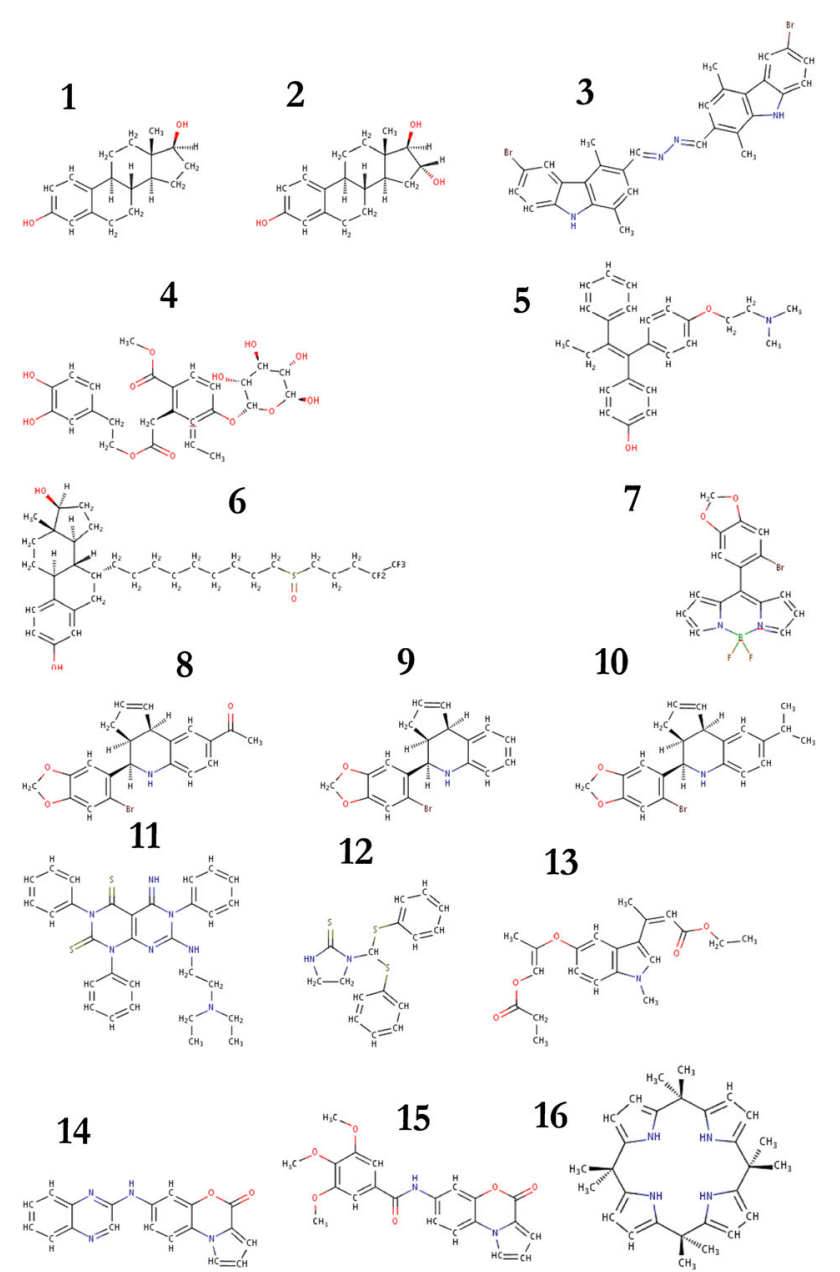

Fig. 1. GPER ligands. 1 17ß-estradiol (E2); 2 estriol (E3); 3 (6bromo-1,4-dimethyl-9H-carbazol-3-yl-methylene)-hydrazine (carbhydraz); 4 oleuropein; 5 4-hydroxytamoxifen (OHT); 6 ICI 182,780(ICI); 7 BODIPY-1; 8 G-1; 9 G-15; 10 G-36; 11 GPER-L1; 12 GPER-L2; 13 Ethyl-3-[5-(2-ethoxycarbonyl-1-methylvinyloxy)-1methyl-1H-indol-3-yl]but-2-enoate (MIBE); 14 7-(Quinoxalin-2ylamino)-4H-benzo[b]pyrrolo[1,2-d][1,4] oxazin-4-one (PBX1) 15 3,4,5-Trimethoxy-N-(4-oxo-4H-benzo[b]pyrrolo[1,2-d][1,4] oxazin-7yl)benzamide (PBX2); 16 calix[4]pyrrole (C4PY)

207, form a disulfide bridge in correspondence of the receptor binding pocket, close to the extracellular side of the protein, then stabilizing the structure.

In our previous studies aimed to design novel selective ligands of GPER and to evaluate the binding modes of known endogenous ligands $(13,27)$, we used a 3D model of GPER obtained by a "supervised" homology modelling. At that time, bovine rhodopsin (38) (PDB Code 1F88) was the best structure available to use as a template, despite the low degree of sequence identity to GPER. Nevertheless, the choice of this template allowed us to build a trustable model of the intramembrane seven-helical bundle. In parallel, the extramembrane $\mathrm{N}$ - and C-terminals together with the EL and IL loops were built $a b$ initio using the servers Robetta (39) and Modeller (40). The resulting model, however, needed some supervised refinement. In particular, the atomic coordinates resulted in a protein structure lacking the disulfide bond between Cys 130 and Cys 207. Hence, in order to achieve a stable and minimized molecular model, a molecular dynamics simulation with energy minimization was mandatory. Once the model had been built, we checked its quality using the software PROCHECK (41). We then decided to perform some molecular docking simulations using both the program suites Gold v.5.2 (the Cambridge Crystallographic Data Center, UK) and Autodock 4.2 (42). The results obtained in silico were confirmed by in vitro experiments, demonstrating a good correspondence between the two procedures (13).

\section{ENDOGENOUS AND EXOGENOUS GPER LIGAND BINDING MODES}

The identification of ligands that may be developed for therapeutic use is the main goal of structure-based drug design (SBDD). Despite the challenges and the problems of docking simulations and the consistency and accuracy of the different scoring functions, virtual screening of molecules through docking simulations leads to different successes.

Using different computational biological techniques, several endogenous and exogenous ligands with agonist or antagonist properties toward GPER have been identified so far by us and other groups (Table I). Following previous studies on the ability of estrogens to activate GPER signalling, a first approach based on computational techniques was adopted by our group with the aim to visualize the binding mode of estriol (E3) (27). Different from the almost similar steroid E2 that acts as a GPER and ER agonist, E3 displays an antagonist action through GPER. The binding mode of E3 involves several hydrophobic and polar residues among which are L137, F206, F208, Y123, Q138, D210, and E275 (Fig. 2a). As it concerns the antiestrogens, tamoxifen (OHT), raloxifene (RAL), and ICI 182,780 (ICI) that are ER antagonists, they behave as agonists toward GPER (43-46). After the identification of GPER as an

Table I. Different Functions of Several GPER Ligands

\begin{tabular}{ll}
\hline Molecule & Activity on GPER \\
\hline 17ß-Estradiol (E2) & Agonist \\
Estriol (E3) & Antagonist \\
4-OHT & Agonist \\
ICI 182,780 & Agonist \\
Oleuropein & Agonist \\
Hydroxytyrosol & Agonist \\
Niacin & Agonist \\
G-1 & Agonist \\
G-15 & Antagonist \\
G-36 & Antagonist \\
MIBE & Antagonist \\
GPER-L1 & Agonist \\
GPER-L2 & Agonist \\
Carbhydraz & Agonist \\
PBX1 & Antagonist \\
PBX2 & Antagonist \\
C4PY & Antagonist
\end{tabular}

C4PY calix[4]pyrrole, E2 17ß-Estradiol, E3 estriol, MIBE Ethyl 3-[5(2-ethoxycarbonyl-1-methylvinyloxy)-1-methyl-1H-indol-3-yl]but-2enoate $O H T$ tamoxifen, $P B X 1$ 7-(Quinoxalin-2-ylamino)-4Hbenzo[b]pyrrolo[1,2-d][1,4]oxazin-4-one, PBX2 3,4,5-Trimethoxy- $N$ (4-oxo-4H-benzo[b]pyrrolo[1,2-d][1,4]oxazin-7-yl)benzamide ${ }^{a}$ MIBE is the only compound today behaving as a "full antagonist" against both GPER and ER 


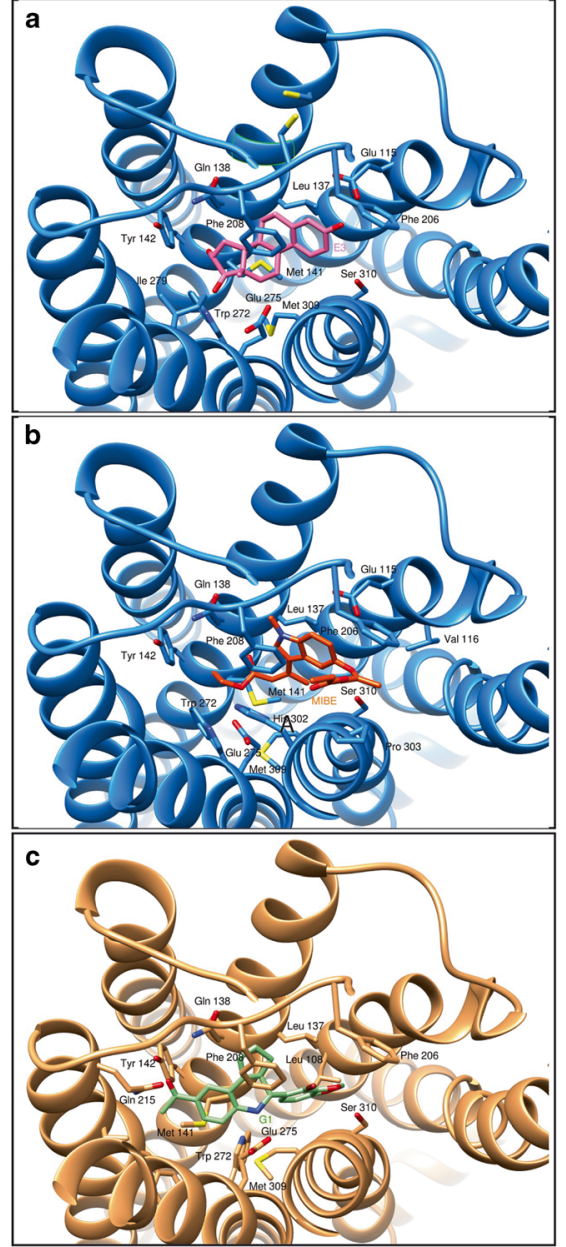

Fig. 2. Ribbon representation of GPER binding sites. Some of the residues involved in ligand binding are drawn as sticks. Estriol (E3) (a) is colored in pink, MIBE is drawn as orange (b), and G-1 in cyan color (c)

alternate estrogen receptor, which can be activated also by antiestrogens, it became necessary to design selective agonist and antagonist ligands in order to elucidate the differential function of GPER with respect to ER. A first success was the identification of the selective agonist chemical G-1 (24), which was obtained by a virtual and biomolecular screening approach. This compound paved the way for the development of further molecules based on the same scaffold, however, exhibiting antagonistic activity as G-15 and G-36 (25,26,28). In parallel, two novel molecules with completely different scaffolds were designed and identified as GPER ligands by docking simulations (29). These two moieties, which were named GPER-L1 and GPER-L2, demonstrated the ability to act as GPER agonists without any affinity for ER. Furthermore, a derivative of the carbazole moiety, named carbhydraz, has been recently synthesized and characterized toward its stimulatory activity through GPER (35). To date, only a molecule exhibited inhibitory properties toward both GPER and ER. This agent named MIBE (30), which was tested in silico using as target $\mathrm{ER} \alpha$, displayed a binding mode similar to that adopted by the ER antagonist OHT in the crystal structure (47) (PDB code 3ERT). When GPER was used as the protein target, MIBE adopted a binding mode which differs from that adopted by G-1 (Fig. 2b, c), although some of the main interactions with the protein are conserved (34). The antagonistic activity toward both GPER and ER displayed by MIBE could strongly suggest a major therapeutic benefit with respect to the antiestrogens currently used in hormone-dependent tumors. Recently, an innovative molecule named $\mathrm{C} 4 \mathrm{PY}$ has been identified as a GPER antagonist (34). C4PY, a macrocyclic compound known as calix[4]pyrrole (molecule 16, Fig. 1), is radically different from any of the molecules adopted in pharmacology to date. To our knowledge, this is the first member of this class of molecules ever adopted as a potential lead compound. Overall, in the last years, several endogenous and exogenous compounds have been screened and their actual ability to bind to GPER determined $(31,32,37,48)$. For instance, two benzopyrroloxazine derivatives, namely PBX1 and PBX2, demonstrated the ability to act as selective GPER antagonists (33). Besides, the rational design and synthesis of the first fluorescent and selective probe of GPER was recently conceived as a BODIPY derivative for bioimaging purposes (49).

\section{CONCLUSIONS}

The multifaceted actions of estrogens are mainly mediated by ER; however, in recent years, increasing evidence have shown that a member of the GPCR family namely GPER may contribute to estrogen signalling in both normal and malignant cells.

Structural studies on GPCRs are very important as many available drugs act by targeting these receptors (50). Unfortunately, these proteins are rather refractory to both X-ray crystallography and NMR techniques for their structure determination, mainly due to the high degree of lipophilicity, the structural plasticity, and the intrinsic difficulties to obtain a rather high-yield expression of recombinant proteins. Computational biology and structural bioinformatics techniques allowed the building of reliable three-dimensional GPCR models. As it concerns GPER, theoretical and computational studies that were performed by structural bioinformatics approaches and molecular modelling gave us the possibility to assess its binding pocket, to simulate the binding modes, and to calculate the affinity constants of different ligands. These findings could be very useful toward a better understanding of the role elicited by estrogenic GPER signalling in diverse pathophysiological conditions.

\section{ACKNOWLEDGMENTS}

This work was supported by Associazione Italiana per la Ricerca sul Cancro (AIRC), PROGRAMMA OPERATIVO NAZIONALE "RICERCA E COMPETITIVITA' 20072013" (PON01_01078) and Ministero della Salute (grant no. 67/GR-2010-2319511).

\section{REFERENCES}

1. Hall JM, Couse JF, Korach KS. The multifaceted mechanisms of estradiol and estrogen receptor signalling. J Biol Chem. 2001;276:36869-72.

2. Selye H. Stress and the general adaptation syndrome. Br Med J. 1950;1:1383-92. 
3. Falkenstein E, Tillmann HC, Christ M, Feuring M, Wehling M. Multiple actions of steroid hormones-a focus on rapid, nongenomic effects. Pharmacol Rev. 2000;52:513-56.

4. Carmeci C, Thompson DA, Ring HZ, Francke U, Weigel RJ. Identification of a gene (GPR30) with homology to the G-protein-coupled receptor superfamily associated with estrogen receptor expression in breast cancer. Genomics. 1997;45:607-17.

5. Prossnitz ER, Hathaway HJ. What have we learned about GPER function in physiology and disease from knockout mice? J Steroid Biochem Mol Biol. 2015;153:114-26.

6. Maggiolini M, Picard D. The unfolding stories of GPR30, a new membrane-bound estrogen receptor. J Endocrinol. 2010;204:105-14.

7. Pandey DP, Lappano R, Albanito L, Madeo A, Maggiolini M, Picard D. Estrogenic GPR30 signalling induces proliferation and migration of breast cancer cells through CTGF. EMBO J. 2009;28:523-32.

8. De Francesco EM, Pellegrino M, Santolla MF, Lappano R, Ricchio E, Abonante S, et al. GPER mediates activation of HIF1 $\alpha$ /VEGF signaling by estrogens. Cancer Res. 2014;74:405364.

9. Madeo A, Maggiolini M. Nuclear alternate estrogen receptor GPR30 mediates 17b-estradiol-induced gene expression and migration in breast cancer-associated fibroblasts. Cancer Res. 2010:70:6036-46.

10. Filardo EJ, Graeber CT, Quinn JA, Resnick MB, Giri D, DeLellis RA, et al. Distribution of GPR30, a seven membranespanning estrogen receptor, in primary breast cancer and its association with clinicopathologic determinants of tumor progression. Clin Cancer Res. 2006;12:6359-66.

11. Smith HO, Leslie KK, Singh MM, Qualls CR, Revankar CM, Joste NE, et al. GPR30: a novel indicator of poor survival for endometrial carcinoma. Am J Obstet Gynecol. 2007;196(386):e11.

12. Smith HO, Arias-Pulido H, Kuo DY, Howard T, Qualls CR, Lee $\mathrm{SJ}$, et al. GPR30 predicts poor survival for ovarian cancer. Gynecol Oncol. 2009;114:465-71.

13. Rosano C, Lappano R, Santolla MF, Ponassi M, Donadini A, Maggiolini M. Recent advances in the rationale design of GPER ligands. Curr Med Chem. 2012;19:6199-206.

14. Berman HM, Westbrook J, Feng Z, Gilliland G, Bhat TN, Weissig $\mathrm{H}$, et al. The Protein Data Bank. Nucleic Acids Res. 2000;28:235-42.

15. Zhang J, Yang J, Jang R, Zhang Y. GPCR-I-TASSER: a hybrid approach to $\mathrm{G}$ protein-coupled receptor structure modeling and the application to the human genome. Structure. 2015;23:1538-49.

16. Bjarnadóttir TK, Gloriam DE, Hellstrand SH, Kristiansson H, Fredriksson R, Schiöth HB. Comprehensive repertoire and phylogenetic analysis of the $G$ protein-coupled receptors in human and mouse. Genomics. 2006;88:263-73.

17. Rosano C. Molecular model of hexokinase binding to the outer mitochondrial membrane porin (VDAC1): implication for the design of new cancer therapies. Mitochondrion. 2011;11:513-9.

18. Clothia C, Lesk AM. The relation between the divergence of sequence and structure in proteins. EMBO J. 1986;5:823-6.

19. Tramontano A. Homology modelling with low sequence identity. Methods. 1998;14:293-300.

20. Kufareva I, Katritch V, Participants of GPCR Dock 2013, Stevens RC, Abagyan R. Advances in GPCR modeling evaluated by the GPCR Dock 2013 assessment: meeting new challenges. Structure. 2013;22:1120-39.

21. Moult J, Fidelis K, Kryshtafovych A, Schwede T, Tramontano A. Critical assessment of methods of protein structure prediction (CASP) - round X. Proteins. 2014;82 Suppl 2:1-6.

22. Arnold K, Kiefer F, Kopp J, Battey JN, Podvinec M, Westbrook JD, et al. The Protein Model Portal. J Struct Funct Genom. 2009;10:1-8.

23. Méndez-Luna D, Martínez-Archundia M, Maroun RC, CeballosReyes G, Fragoso-Vázquez MJ, González-Juárez DE, et al. Deciphering the GPER/GPR30-agonist and antagonists interactions using molecular modeling studies, molecular dynamics, and docking simulations. J Biomol Struct Dyn. 2015;33:2161-72.

24. Bologa CG, Revankar CM, Young SM, Edwards BS, Arterburn $\mathrm{JB}$, Kiselyov AS, et al. Virtual and biomolecular screening converge on a selective agonist for GPR30. Nat Chem Biol. 2006;4:207-12.
25. Dennis MK, Burai R, Ramesh C, Petrie WK, Alcon SN, Nayak TK, et al. In vivo effects of a GPR30 antagonist. Nat Chem Biol. 2009;5:421-7.

26. Ramesh C, Nayak TK, Burai R, Dennis MK, Hathaway HJ, Sklar LA, et al. Synthesis and characterization of iodinated tetrahydroquinolines targeting the $\mathrm{G}$ protein-coupled estrogen receptor GPR30. J Med Chem. 2010;53:1004-14.

27. Lappano R, Rosano C, De Marco P, De Francesco EM, Pezzi V, Maggiolini M. Estriol acts as a GPR30 antagonist in estrogen receptor-negative breast cancer cells. Mol Cell Endocrinol. 2010;320:162-70.

28. Dennis MK, Field AS, Burai R, Ramesh C, Petrie WK, Bologa $\mathrm{CG}$, et al. Identification of a GPER/GPR30 antagonist with improved estrogen receptor counterselectivity. J Steroid Biochem Mol Biol. 2011;127:358-66.

29. Lappano R, Rosano C, Santolla MF, Pupo M, De Francesco EM, De Marco P, et al. Two novel GPER agonists induce gene expression changes and growth effects in cancer cells. Curr Cancer Drug Targets. 2012;12:531-42.

30. Lappano R, Santolla MF, Pupo M, Sinicropi MS, Caruso A, Rosano $\mathrm{C}$, et al. MIBE acts as antagonist ligand of both estrogen receptor $\alpha$ and GPER in breast cancer cells. Breast Cancer Res. 2012;14:R12.

31. Santolla MF, De Francesco EM, Lappano R, Rosano C, Abonante S, Maggiolini $M$. Niacin activates the $G$ protein estrogen receptor (GPER)-mediated signalling. Cell Signal. 2014;26:1466-75.

32. Chimento A, Casaburi I, Rosano C, Avena P, De Luca A, Campana $\mathrm{C}$, et al. Oleuropein and hydroxytyrosol activate GPER/ GPR30-dependent pathways leading to apoptosis of ER-negative SKBR3 breast cancer cells. Mol Nutr Food Res. 2014;58:478-89.

33. Maggiolini M, Santolla MF, Avino S, Aiello F, Rosano C, Garofalo A, et al. Identification of two benzopyrroloxazines acting as selective GPER antagonists in breast cancer cells and cancer-associated fibroblasts. Future Med Chem. 2015;7:437-48.

34. Lappano R, Rosano C, Pisano A, Santolla MF, De Francesco EM, De Marco P, et al. A calixpyrrole derivative acts as a GPER antagonist: mechanisms and models. Dis Model Mech. 2015. doi:10.1242/dmm.021071.

35. Sinicropi MS, Lappano R, Caruso A, Santolla MF, Pisano A, Rosano C, et al. (6-bromo-1,4-dimethyl-9H-carbazol-3-yl-methylene)-hydrazine (carbhydraz) acts as a GPER agonist in breast cancer cells. Curr Top Med Chem. 2015;15:1035-42.

36. Owman C, Blay P, Nilsson C, Lolait SJ. Cloning of human cDNA encoding a novel heptaelix receptor expressed in Burkitt's lymphoma and widely distributed in brain and peripheral tissues. Biochem Biophys Res Commun. 1996;228:285-92.

37. Prossnitz ER, Arterburn JB. International Union of Basic and Clinical Pharmacology. XCVII. G protein-coupled estrogen receptor and its pharmacologic modulators. Pharmacol Rev. 2015;67:505-40.

38. Palczewski K, Kumasaka T, Hori T, Behnke CA, Motoshima H, Fox BA, et al. Crystal structure of rhodopsin: a G proteincoupled receptor. Science. 2000;289:739-45.

39. Kim DE, Chivian D, Baker D. Protein structure prediction and analysis using the Robetta server. Nucleic Acids Res. 2004;32(Web Server issue): W526-W531.

40. Sali A, Blundell TL. Comparative protein modelling by satisfaction of spatial restraints. J Mol Biol. 1993;234:779-815.

41. Laskowski RA, MacArthur MW, Moss DS, Thornton JM. PROCHECK - a program to check the stereochemical quality of protein structures. J App Crystallogr. 1993;26:283-91.

42. Morris GM, Huey R, Lindstrom W, Sanner MF, Belew RK, Goodsell DS, et al. Autodock4 and AutoDockTools4: automated docking with selective receptor flexiblity. J Comput Chem. 2009;16:2785-91.

43. Filardo EJ, Quinn JA, Bland KI, Frackelton Jr AR. Estrogeninduced activation of Erk-1 and Erk-2 requires the G proteincoupled receptor homolog, GPR30, and occurs via transactivation of the epidermal growth factor receptor through release of HB-EGF. Mol Endocrinol. 2000;10:1649-60.

44. Revankar CM, Cimino DF, Sklar LA, Arterburn JB, Prossnitz ER. A transmembrane intracellular estrogen receptor mediates rapid cell signalling. Science. 2005;307:1625-30. 
45. Vivacqua A, Bonofiglio D, Recchia AG, Musti AM, Picard D, Andò $\mathrm{S}$, et al. The $\mathrm{G}$ protein-coupled receptor GPR30 mediates the proliferative effects induced by $17 \beta$-estradiol and hydroxytamoxifen in endometrial cancer cells. Mol Endocrinol. 2006;20:631-46.

46. Vivacqua A, Bonofiglio D, Albanito L, Madeo A, Rago V, Carpino A, et al. 17ß-Estradiol, genistein, and 4hydroxytamoxifen induce the proliferation of thyroid cancer cells through the G protein coupled-receptor GPR30. Mol Pharmacol. 2006;70:1414-23.

47. Shiau AK, Barstad D, Loria PM, Cheng L, Kushner PJ, Agard $\mathrm{DA}$, et al. The structural basis of estrogen receptor/coactivator recognition and the antagonism of this interaction by tamoxifen. Cell. 1998;95:927-37.

48. Albini A, Rosano C, Angelini G, Amaro A, Esposito AI, Maramotti S, et al. Exogenous hormonal regulation in breast cancer cells by phytoestrogens and endocrine disruptors. Curr Med Chem. 2014;21:1129-45.

49. Papalia T, Lappano R, Baratucci A, Pisano A, Bruno G, Santolla MF, et al. A Bodipy as a luminescent probe for detection of the $\mathrm{g}$ protein estrogen receptor (GPER). Org Biomol Chem. 2015. doi:10.1039/C5OB01827G.

50. Lappano R, Maggiolini M. G protein-coupled receptors: novel targets for drug discovery in cancer. Nat Rev Drug Discov. 2011;10:47-60. 